\title{
Jan Potocki et son Manuscrit trouvé à Saragosse : quelques interrogations d'un russisant
}

Leonid Heller

\section{(2) OpenEdition \\ 1 Journals}

Édition électronique

URL : http://journals.openedition.org/edl/460

DOI : $10.4000 /$ edl. 460

ISSN : 2296-5084

Éditeur

Université de Lausanne

\section{Édition imprimée}

Date de publication : 15 décembre 2012

Pagination : 115-132

ISBN : 978-2-940331-29-1

ISSN : 0014-2026

Référence électronique

Leonid Heller, « Jan Potocki et son Manuscrit trouvé à Saragosse : quelques interrogations d'un russisant », Études de lettres [En ligne], 4 | 2012, mis en ligne le 15 décembre 2015, consulté le 18 décembre 2020. URL : http://journals.openedition.org/edl/460 ; DOI : https://doi.org/10.4000/edl.460 


\section{JAN POTOCKI ET SON MANUSCRIT TROUVÉ À SARAGOSSE: QUELQUES INTERROGATIONS D'UN RUSSISANT}

Si dans les études russes, Potocki et son roman prennent une certaine place, cela se passe avant tout sur le terrain de la pouchkinologie. Le présent article tente d'élargir la perspective en interrogeant le contexte historique et la possible filiation potockienne dans la littérature russe. Outre les grands auteurs, Gogol ou Lermontov, de nombreux romanciers exploitent, à côté des ambiances fantastiques, le principe narratif de l'arabesque avec son entrelacs de voix narratrices et de formules génériques, proche du modèle d'écriture potockien. Le parcours proposé permet de préciser les caractéristiques de ce dernier, à entrevoir l'«anti-sternianisme» du Manuscrit et à repérer un manque significatif dans ce «roman somme», celui de l'utopie.

Pour commencer, j'aimerais dire ma reconnaissance d'avoir été invité à ce colloque ${ }^{1}$. Je connaissais le Manuscrit trouvé à Saragosse depuis assez longtemps, comme une évidence; il oscillait à la périphérie de mon champ d'études. Grâce à cette invitation, j’ai compris ma coupable négligence et aussi l'ampleur de la redécouverte quapporte la nouvelle édition des deux versions du roman. Exotisme, orientalisme, fantastique, narration romanesque, pour tous ces domaines que j'aime fréquenter, Potocki s'avère incontournable, même pour un russisant.

Un mot en guise de préambule. La littérature «potockologue» est bien abondante; je n’ai pu la maîtriser. Je ne garantis donc pas l'originalité

I. La rédaction n'a pas jugé nécessaire d'atténuer les signes d'oralité qui abondent dans ce texte, transcription de l'intervention prononcée lors de la journée d'étude du 27 janvier 2010 à Lausanne. 
de mes observations. Exemple: à la relecture du Manuscrit, il m'a paru clair que le film de Luis Buñuel La Voie lactée (1969), que je tiens pour un chef-d'œuvre du modernisme déconstruit, y renvoie par sa mosaïque d'éléments: voyage, onirisme, présence protéiforme du diable, libertinage, débats sur la foi, questionnements philosophiques, récit éparpillé en épisodes, mélange de tonalités et de styles unifié par une distanciation ironique. Dans les articles que j'ai lus sur Potocki, je n'ai pas vu faire ce rapprochement qui, pour moi, vaut une bonne piste à la fois pour une analyse typologique et pour une enquête sur la place de Potocki dans notre présent multiculturel et "multimédial». Mais mon observation est-elle vraiment nouvelle? Autre exemple: orthographié à l'espagnole, le nom Zaragoza laisse lire les mots polonais groza et zgroza: «épouvante» et "sentiment d'horreur». Le Manuscrit est bien - entre autres - un roman d'épouvante. A-t-on déjà fait cette lecture? Elle ne contredit nullement la référence quichottesque qu'avance François Rosset ${ }^{2}$; elle l'enrichit d'un complément symbolique, intentionnel ou inconscient, dû au "génie de la langue", peu importe. Néophyte, je demande donc d'excuser le peu de rigueur de mon exposé, construit autour de plusieurs interrogations qui risquent de concerner des vérités établies depuis longtemps.

J'ai cependant une excuse. MM. Rosset et Triaire considèrent très justement le Manuscrit comme un "roman somme», un «roman bilan" ${ }^{3}$. L'étude d'une telle œuvre reste en permanence ouverte et incite à établir, pour mieux la voir, des points d'observation toujours nouveaux. C'est pour cette raison que j'ose présenter ici mes propositions en espérant, à terme, qu'une perspective se mette en place dans laquelle le contexte russe et Potocki pourraient s'éclairer mutuellement.

On sait que des épisodes importants lient la carrière et l'œuvre de Potocki à la Russie; or peu de travaux de trois côtés - occidental, polonais, russe - explorent ce lien autrement que par le biais biographique. On résumera l'état des recherches sans perdre de vue que les conditions d'investigation et de publication diffèrent entre les aires culturelles; de plus, ces conditions ont beaucoup évolué avec le temps.

On connaît l'article de Neil Cornwell qui a tenté d'élargir le contexte de réception littéraire du Manuscrit jusqu'à y inclure la littérature russe

2. F. Rosset, «Pourquoi Saragosse?».

3. Cf. F. Rosset, D. Triaire (éds), De Varsovie à Saragosse et "Présentation"; F. Rosset, «Roman grotesque parce que roman-somme». 
et, plus particulièrement, le roman de Vladimir Odoïevski Les Nuits russes $(1844)^{4}$. Dans une thèse de doctorat récente, Svetlana SamokhinaTrouvé voit en Potocki le précurseur de ce mode d'écriture ${ }^{5}$; elle énonce, mais sans la creuser vraiment, cette idée qui semble extrêmement productive: je vais y revenir. Si d'autres travaux occidentaux sur la présence potockienne dans la culture russe existent, j’avoue les ignorer.

Dans les études russes, l'époque soviétique a imposé une vision particulière autant des Lumières et du XIX ${ }^{e}$ siècle que des échanges et des «transferts culturels» entre la Russie et la Pologne, la Russie et le reste de l'Europe. Cette vision «marxiste-progressiste" s'accommode mal de la figure de Potocki. Pendant longtemps, son nom est resté sinon tabou, du moins peu recommandé et peu évoqué. Ni l'Encyclopédie littéraire (Literaturnaja ènciklopedija) de 1935, ni les Encyclopédies Soviétiques Petite et Grande jusque dans les années 1980 n'en parlent. L'ouverture a lieu en 1968; l'Encyclopédie littéraire accorde à Potocki une petite notice et la même année le Manuscrit est pour la première fois publié en russe, dans la prestigieuse série "Monuments littéraires", traduit par Aleksandr Golemba et préfacé par Igor Belza, musicologue et connaisseur des cultures slaves ${ }^{6}$. En 1971, une autre traduction voit le jour, de Dmitri Gorbov, avec une préface circonstanciée de Sémion Landa, historien spécialiste de l'époque décembriste 7 . Des rééditions suivent (au moins sept dans les années 1990 : témoignage d'un réel succès auprès des lecteurs). Les préfaces de Belza et Landa sont recyclées, reprises, citées, notamment dans divers ouvrages collectifs ${ }^{8}$; mais d'autres travaux tardent à venir. Si bien qu'il faut, pour compléter la liste, mentionner ici quelques chercheurs des années 1920-1930: Vladimir Frantsev, Iouri Tomachevski, Viktor Tchernobaev. On s'aperçoit bien vite que l'inspiration principale et la charpente de toutes ces études sont constituées par un seul grand thème, central pour la culture russe et définitivement sacralisé par la culture soviétique: le thème pouchkinien.

4. N. Cornwell, "The European tradition of the "Nights" ".

5. Svetlana Samokhina-Trouvé, Le fantastique dans la prose romantique russe, Thèse de doctorat, Paris IV-Sorbonne, 2007.

6. J. Potockij, Rukopis', najdennaja v Saragose.

7. I. Bèlza, «Puškin i pol'skaja kul'tura», "Puškin i Jan Potockij» et Portrety romantykow; S. Landa, "Jan Potockij i ego roman Rukopis", najdennaja v Saragose» et "Ja vižu nekij svet...".

8. S. Landa, "Jan Potockij i ego roman Rukopis', najdennaja v Saragose». 
C'est que Pouchkine lui-même, dans son récit-reportage de la campagne turque, Voyage à Arzroum (1829), dit s'être inspiré des écrits de voyages de Potocki, "aussi passionnants que ses romans espagnols"; le grand poète russe possède dans sa bibliothèque les deux éditions parisiennes partielles du Manuscrit ${ }^{9}$; vers 1835, il compose une "variation potockienne", le poème devenu célèbre "Alphonse enfourche son coursier " ${ }^{10}$. L'intérêt pour Potocki se trouve ainsi légitimé; les chercheurs peuvent aller au-delà de la scène initiale pouchkinienne et décrire, avec plus ou moins de détails, l'œuvre et la vie de cet "aristocrate excentrique». Ils abordent toutefois sans témérité ce qui concerne la possible diffusion de l'influence potockienne dans la littérature russe.

En Pologne, la situation est bien entendu différente. Depuis le $\mathrm{XIX}^{\mathrm{e}}$ siècle, on y publie et étudie celui qu'Adam Mickiewicz a appelé, dans son cours au Collège de France, "un des écrivains les plus célèbres et les plus profonds de la Pologne» ${ }^{11}$.

Le Manuscrit est publié à Varsovie, dans la traduction classique de Chojecki, sous le "haut stalinisme» en 1950, pendant le Dégel poststalinien, et lors de la libéralisation du régime ${ }^{12}$. Il a été observé que le renouveau d'intérêt, dans les années 1960-1970, pour le roman et son auteur avait stimulé une réévaluation plus générale de la culture polonaise du XVIII siècle $^{13}$. Potocki est devenu une sorte de figure tutélaire du courant anti-«réaliste socialiste»; la fameuse adaptation cinématographique que Wojciech Has tourne en 1964 confirme brillamment cette réputation. Mais, dans une Pologne socialiste, l'analyse des relations avec la Russie reste difficile, même lorsque la vision "marxiste-progressiste» perd de son caractère contraignant. On évoquera ici Andrzej Dworski, jeune pouchkiniste décédé en 1975; son remarquable recueil d'études, paru seulement en 1983, huit ans après sa mort, inclut un texte riche en

9. Avadoro, histoire espagnole et Dix journées d'Alphonse van Worden, publiés respectivement en 1813 et 1814 par le libraire Gide.

Io. "Alfons saditsja na konja". Le poème figure dans la plupart des éditions, académiques ou populaires, de Pouchkine. Voir le texte en russe et sa traduction littérale en annexe.

II. A. Mickiewicz, «Le cours du 15 mars 1842», p. 206.

I2. J. Potocki, Rẹkopis znaleziony w Saragossie, [Warszawa], Czytelnik, 1950, 1956, 1965 et 1976.

I3. I. Bèlza, "O badaniach polskiej kultury artystycznej XVIII-XIX stulecia», p. 20. 
détails sur "Pouchkine et Jan Potocki» ${ }^{14}$. Ce recueil sera publié en russe, en 1999, fruit d'un transfert réciproque suffisamment rare pour être noté $^{15}$. Sinon, c'est encore à Igor Belza, traduit du russe, qu'il revient de parler en polonais de Potocki en Russie; il le fait sans jamais trop s'éloigner des rivages pouchkiniens ${ }^{16}$.

Bref, les travaux sur le sujet qui nous intéresse, les relations entre Potocki et son héritage avec la littérature russe, sont peu nombreux et couvrent peu de terrain. On peut en tirer cependant une information essentielle: dans les années 1820-1830, Potocki est bien connu de l'élite pétersbourgeoise. Son roman marque les esprits. On raconte comment Potocki l'invente des nuits durant pour amuser sa femme malade. On diffuse des légendes sur son suicide ${ }^{17}$. Le Journal de Philippe Wiegel, un ami de Pouchkine, consigne son portrait extravagant ${ }^{18}$. Le prince Pierre Viazemski, un autre ami de Pouchkine et une bonne connaissance de Seweryn Potocki, frère de Jean, écrit en 1826, l'année qui suit la malheureuse révolte décembriste, terminée par la pendaison publique de ses cinq meneurs :

Je me sens comme ce héros de Potocki qui, où qu'il soit, quoi qu'il fasse, se réveille toujours sous une potence: et moi, quelles que soient mes pensées ou mes occupations, malgré moi et d'une manière inattendue, je me retrouve toujours au pied de ces cinq abominables gibets qui ont transformé pour moi la Russie en un effroyable lieu d'exécution ${ }^{19}$.

Mais les adversaires littéraires et politiques de Pouchkine apprécient également Potocki, tel le journaliste et auteur prolifique Thaddée Boulgarine, qui publie dans sa revue, en 1828, un fragment du Voyage dans les steps d'Astrakhan..., ou bien l'historien et philologue Nikolaï Gretch, qui écrit, en 1825 :

14. A. Dworski, «Jan Potocki i Puszkin».

15. A. Dvorskij, "Jan Potockij i Puškin».

I6. P. ex., I. Bèlza, "O badaniach polskiej kultury artystycznej XVIII-XIX stulecia».

I7. Cf. V. Černobaev, "K istorii nabroska "Al'fons saditsja na konja”".

I8. F. Vigel', Zapiski.

19. P. Viazemski, lettre à sa femme, 1826, cité d'après K. Galon-Kurkowa, Dlugi zmierzch, p. 76. 
Les amateurs de l'histoire et de la littérature vont longtemps pleurer le départ intempestif de ce savant, cet écrivain sage, doté de tous les talents. Je garde comme un bijou précieux son insolite roman Le Manuscrit trouvé à Saragosse, commencé devant le mur de la Sierra Morena et terminé au pied de la Grande Muraille chinoise! Il n’a édité que cinquante exemplaires de cette œuvre en caractères italiques, sans titre, sans nombre de pages, sans fin ${ }^{20}$.

On peut probablement parler d'un "mythe Potocki», né à cette époque, colporté discrètement dans les milieux maçonniques et polonais de la capitale, et diffusé plus loin, jusqu'aux marches de l'Empire, dans ces espaces indécidables entre la Russie et la Pologne, entre l'Europe et l'Orient, où l'écrivain et géographe avait sa demeure, qu'il traversait sans cesse et qu'il a choisis pour mourir.

Ce mythe continue à vivre: au début des années 1980, le poète russoukrainien se réclamant des ancêtres polonais Viktor Sosnora écrit un poème qui exploite le thème du suicide avec une balle d'argent et qui devient connu au point d'être mis en musique par plusieurs chanteurs de rock. Dans ce poème, un critique d'aujourd'hui voit une référence explicite au mythe de Potocki ${ }^{21}$.

Revenons aux interrogations posées au début de cet article. En voici une, d'ordre biographique; elle concerne les manœuvres de Potocki pour avoir l'oreille du pouvoir à Pétersbourg. On a écrit qu'il cherchait des appuis auprès du favori de Catherine II, Platon Zoubov ${ }^{22}$. Il n'est peut-être pas hors de propos de préciser que ledit Zoubov est resté dans l'histoire surtout en tant quauteur du fameux «projet grec» qui devait subrepticement inspirer la politique de l'impératrice. Il redessinait une Europe d'avenir, après la victoire définitive sur le rival ottoman: il ne devait plus y avoir de Pologne, d'Autriche, de Hongrie, seulement un vaste continent russe doté de six capitales: Moscou, Pétersbourg, Varsovie, Vienne, Berlin et enfin Constantinople, la Ville sacrée des Tsars: le Tsargrad. Potocki cherchait une aide logistique et financière pour ses projets scientifiques; pensait-il, espérait-il que sa connaissance de la Turquie et, plus généralement, son expertise ès matières orientales

20. Texte de 1825, cité d'après A. Dworski, «Jan Potocki i Puszkin», p. 212.

2I. Cf. E. Slivkin, «Stixotvorenie, najdennoe v Saragosse».

22. Voir par exemple F. Rosset, D. Triaire, «Présentation», p. 19. 
pouvaient être utiles pour le projet de Zoubov? La réponse à cette question éclaircirait sa position vis-à-vis du pouvoir de Pétersbourg.

A un autre moment, Potocki obtient l'accès à Paul $\mathrm{I}^{\mathrm{er}}$; il semble que ses tentatives d'obtenir le soutien de l'empereur se soldent par un échec: la mort soudaine de Stanislas Auguste, ancien roi de Pologne et protecteur de Potocki, en serait responsable. Or Potocki a été chevalier de l'Ordre de Malte, dont Paul I ${ }^{\text {er }}$ venait d'être nommé le grand Maître. Belza décrit, sans citer ses sources, une audience que l'empereur aurait accordée à Potocki, au milieu de symboles de l'ordre maltais ${ }^{23}$. Ce lien entre les deux hommes aurait attiré, dit Belza, l'attention de Pouchkine qui faisait une recherche sur l'assassinat de Paul, perpétré avec l'accord tacite de son fils, futur Alexandre I ${ }^{\text {er }}$ (et il faut dire qu'il y a eu des chevaliers de Malte parmi les assassins). On imagine le genre de questions "romanesques" que cet épisode peut inciter à poser. Plus concrètement, il me semble que la piste maltaise mérite d'être suivie de près; elle peut conduire à la fois sur des versants peu explorés de la vie de Potocki, vers des secrets de sa création et renseigner aussi sur les canaux de la diffusion de celle-ci.

Autre nœud problématique: le Manuscrit en tant que source d'inspiration et/ou matière d'exploitation pour plusieurs générations d'écrivains russes. La thèse déjà mentionnée sur la littérature fantastique suggère que le roman de Potocki participe, préside même, à sa formation et à son évolution. Cette hypothèse que je partage, du moins en partie, permet d'affirmer la nécessité de relire sous cet angle les auteurs du $\mathrm{XIX}^{\mathrm{e}}$ siècle, en essayant de ne pas tomber dans le piège d'analogies trop directes. J'ai effectué plusieurs sondages qui me permettent de formuler quelques observations au moins liminaires.

On ne reviendra pas sur Pouchkine. Mais prenons Gogol: ses inclinations orientalistes, ses tentations diaboliques, son goût du pittoresque et surtout sa poétique qui joue sur l'épouvante, qui fait se télescoper le sublime et le comique, se rapprochent de la démarche potockienne, mais n'autorisent pas de conclure à quelque influence directe. Ici, E. T. A. Hoffman et les «frénétiques» français pourraient suffire comme référence (mais Jules Janin ne marchait-il pas dans les pas de Potocki ?). En revanche, la manière stylistique de Gogol permet de trouver une analogie à la structure narrative enchâssée du Manuscrit:

23. I. Bèlza, «Puškin i Jan Potockij», p. 130 sq. 
son principe semble correspondre à celui de l'arabesque qui organise divers motifs en les répétant et en les entrecroisant. Si bien que les motifs disparaissent et réapparaissent à nouveau et que la distinction entre le fond et la forme s'efface, l'arrière-plan et le premier plan se confondent. L'arabesque est une des figures essentielles de l'esthétique romantique; il me paraît incontestable que sur le plan narratif, cette figure est réalisée d'une manière spectaculaire et exemplaire dans le Manuscrit.

Prenons Lermontov; on ne l'a jamais étudié de ce point de vue. Or d'une part, il fréquentait des cercles, des cénacles dont faisaient partie des aristocrates polonais, tel Ksawery Branicki, qui ne pouvaient pas ne pas connaître Potocki ${ }^{24}$. Est-ce suffisant pour décréter que certaines scènes du Héros de notre temps (1841), certains personnages, la sauvagerie des paysages, la violence du récit mâtinée d'un humour froid, les jeux de focalisation et de voix narratives, la multiplicité de formules génériques et topiques, allant du récit de voyage au roman de pirates et d'épouvante, que tout cela fait penser au Manuscrit? Là encore, c'est une piste qu'il faudrait suivre de près.

Continuons un moment sur notre lancée. Nous l'avons déjà vu, un parallèle tout à fait justifié a été établi entre Potocki et Vladimir Odoïevski, auteur essentiel pour le romantisme russe. Or, il y a autant de raisons de chercher des points communs entre le modèle potockien et d'autres écrits romanesques, fantastiques, comiques qui paraissent en Russie tout au long du XIX ${ }^{\mathrm{e}}$ siècle. Qu'il suffise d'évoquer ici le Polonais renégat Thaddée Boulgarine (Tadeusz Bulharyn), auteur de romans d'aventures, best-sellers de l'époque; le fantasque "Baron Brambeus", nom de plume d'Ossip (Jozef) Senkowski, orientaliste célèbre, également Polonais; Alexandre Veltman, un savant archaïsant et un romancier novateur. J'éviterai d'ennuyer le lecteur non prévenu en alignant des noms connus des seuls spécialistes de la littérature russe. Il faut cependant ajouter à cette liste trop brève deux auteurs qui appartiennent déjà à l'époque suivante. Dans un poème de Konstantin Sloutchevski, précurseur du symbolisme, écrit en 1878 , le cauchemar du héros qui avait vu un criminel exécuté à Genève et qui s'imagine être une corde de guitare malmenée par une sorcière, rappelle curieusement le récit des tortures du pauvre démoniaque Pacheco. Tandis que L’Ange de feu (1907) de Valéry Brioussov, chef de file du mouvement symboliste russe, est un roman

24. Cf. E. Gerštejn, «Lermontov i kružok šesnadcati». 
fantastique situé en Allemagne au XVI ${ }^{\mathrm{e}}$ siècle et qui semble utiliser un certain nombre d'éléments potockiens: ainsi, un mariage rêvé avec un être surnaturel, tout comme dans le récit de Rebecca, des illusions diaboliques, des rencontres et discussions avec des cabalistes, et finalement le personnage central du héros lui-même, un chevalier courageux et distancié, qui traverse le monde en observateur. Brioussov joue sur la diversité de tonalités et sur de multiples confrontations entre la rationalité et la magie, l'onirisme et le commentaire scientifique; il présente son récit comme un manuscrit d'époque égaré et retrouvé par hasard, bref, c'est exactement comme s'il s'inspirait de l'écrivain polonais.

Mes intuitions doivent être vérifiées et revérifiées: si elles se confirmaient, on obtiendrait le tableau d'une impressionnante postérité de Potocki dans le fantastique russe. L'étude de celle-ci permettrait à son tour de préciser les traits les plus pertinents du modèle de l'écriture potockienne.

Pour donner un peu plus de consistance à ce point, j'aimerais revenir à un auteur déjà nommé, Alexandre Veltman. On sait de lui que, dans les années 1830, il cherchait auprès de ses amis à se procurer des écrits de Potocki ${ }^{25}$. Avec ce dernier, Veltman partage son amour pour l'archéologie, l'histoire et la géographie; il dépasse Potocki dans sa propension à mettre son savoir au service d'une mythologie, tant personnelle que «nationale». Veltman va écrire quelques-uns parmi les romans russes les plus curieux, dont certains utilisent déjà le procédé de voyage dans le temps pour parler du passé historique avec une liberté débridée et réjouissante (ainsi, il a tenté de démontrer, aussi bien dans ses fictions romanesques que dans ses travaux prétendument scientifiques, les origines slaves d'Alexandre le Grand) ${ }^{26}$. Son premier roman s'intitule Le Pélerin (ou Le Voyageur, 1831); un carnet de voyage qui inclut des récits d'aventures enchâssés, des poèmes, des dialogues philosophiques, des fragments de récit de guerre, des scènes historiques (telle la mort d'Alexandre le Grand) ${ }^{27}$. Cette profusion générique et cette liberté narrative rappellent bien entendu le Manuscrit. On pourrait dire toutefois que Veltman conçoit son roman comme une sorte d'anti-Manuscrit.

25. S. Landa, "Jan Potockij i ego roman...", p. 33.

26. Cf. L. Heller, M. Niqueux, Histoire de l'utopie en Russie, p. 178.

27. A. Vel'tman, Strannik. 
En parlant de la passion de voyageur qui consumait Potocki et de ses multiples déplacements, Daniel Beauvois a écrit:

Sans cette fièvre de voyage le mouvement caléïdoscopique du Manuscrit n'existerait pas et les nombreuses mises en abyme de voyage dans le voyage ne résoudraient pas la difficulté, pour le narrateur, de tout voir de ses propres yeux ${ }^{28}$.

Le pèlerin excentrique de Veltman donne tort à cette idée; il parcourt le monde sans se déplacer, en restant assis devant une carte géographique; il suit un parcours fantasque, dévié parfois par un pâté d'encre qui tombe de sa plume, et se souvient des histoires lues et entendues sur différents lieux qu'il traverse, et en invente d'autres. Bref, il reprend à son compte la critique ironique que Laurence Sterne adressait à la mode de voyager partout sans rien voir, dans la préface à son Voyage sentimental. Veltman est sternien et, dans son roman, il suit l'exemple d'un autre sternien, Xavier de Maistre dont Voyage autour de ma chambre date de 1794, l'année même où Potocki s'est mis à composer son Manuscrit. Je m'étonne de n'avoir trouvé, dans les articles que j'ai pu lire sur le roman de Potocki, qu'une très vague mention de son sternianisme ${ }^{29}$. Or, on sent bien Sterne dans les pages du Manuscrit, dans ses personnages, tous dotés de manies spécifiques et cocasses, dans des passages qui évoquent clairement les discussions de l'oncle Toby avec le caporal Trim:

Mon père s'établit de l'autre côté du salon sur des tables jointes par des planches et, de son lit à celui de ma mère, on pratiqua une jetée fortifiée dans le milieu par une sorte de batardeau, construit de coffres et de caisses. Cet ouvrage fut achevé le jour même de notre arrivée au château, et je suis venu au monde neuf mois après, jour pour jour. Note: A la lecture de ces lignes burlesques, on se rappelle que Potocki avait été nommé lieutenant dans le génie... ${ }^{30}$

Il y a, entre deux formes de voyages, réel et mental, une tension réciproque formatrice: l'une n'existe pas sans l'autre. De Maistre vantait ainsi sa pratique de voyage immobile:

\footnotetext{
28. D. Beauvois, "Jean Potocki's Voyages», p. 16.

29. M. Żurowski, "Le Manuscrit trouvé à Saragosse et la technique romanesque du XVIII" s.", p. 110 sq.

30. MTS-1804, p. 108. La note citée provient des éditeurs.
} 
Depuis l'expédition des Argonautes jusqu'à l'assemblée des Notables ; depuis le fin fond des enfers jusqu'à la dernière étoile fixe au-delà de la voie lactée, jusqu'aux confins de l'univers, jusqu'aux portes du chaos, voilà le vaste champ où je me promène en long et en large, et tout à loisir; car le temps ne me manque pas plus que l'espace. C'est là que je transporte mon existence à la suite d'Homère, de Milton, de Virgile, d'Ossian, etc. ${ }^{31}$

La fièvre de déplacements, de découvertes exotiques, de recherches de l'Autre, de l'expatriation, a d'autant plus de sens dans la littérature romanesque qu'elle s'y confronte et se mélange en permanence à son contraire et qu'une place si importante y est occupée par Sterne. Je n'aurais pas remarqué ce côté "anti-Sterne" chez Potocki sans être passé par l'«antipotockisme» d'Alexandre Veltman et par le sternianisme russe.

Je conclus en revenant à la définition du roman de Potocki comme «somme»- pour constater un manque qui me semble important et qui mériterait qu'on s'y arrête plus minutieusement qu'il ne m'est possible de le faire ici: j'entends le manque de l'utopie. Faut-il rappeler que le XVIII ${ }^{e}$ siècle fut un siècle utopique et qu'il a largement été suivi dans ce domaine par le siècle suivant. Le passage le plus proche de l'utopie dans le Manuscrit est présenté, me semble-t-il, dans l'Histoire du marquis de Val Florida:

La vertu du jeune Sidonia était son idole, le bien public sa chimère. Nous faisions une étude particulière de la constitution de l'Espagne et beaucoup de plans pour sa prospérité future; pour rendre les Espagnols heureux, nous voulions d'abord leur faire aimer la vertu et ensuite les détacher de leur intérêt, ce qui nous paraissait très facile. Nous voulions aussi ranimer l'antique esprit de chevalerie. [...] Ainsi livrant notre imagination à de vertueux écarts, le duc et moi, nous espérions réaliser en Espagne les règnes de Saturne et de Rhée, mais pendant ce temps-là, Van Berg y ramenait réellement l'âge d'or ${ }^{32}$.

On voit déjà qu'il s'agit là d'une parodie; on sait qu'elle va se transformer en un drame sanglant. Potocki le pessimiste s'éloigne des préceptes maçonniques; il n'accepte pas d'utopie dans son monde. Par là encore il rejoint Xavier de Maistre qui écrivait:

31. X. de Maistre, Voyage autour de ma chambre, p. 155.

32. MTS-1810, p. 371 sq. 
Nous étions heureux par nos erreurs. - Et maintenant:- Ah! ce n'est plus cela; il nous a fallu lire, comme les autres, dans le cœur humain; - et la vérité, tombant au milieu de nous comme une bombe, a détruit pour toujours le palais enchanté de l'illusion ${ }^{33}$.

\section{Leonid Heller}

Université de Lausanne

33. X. de Maistre, Voyage autour de ma chambre, p. 146. 


\section{ANNEXE}

Poème de A. Pouchkine «Alphonse enfourche son coursier" (vers 18351836, publication posthume dans les Euvres, 1841)

Al'fons saditsja na konja;

Emu xozjain deržit stremja.

„Sen'or, poslušajtes' menja:

Puskat'sja v put' teper' ne vremja,

V gorax opasno, noč blizka,

Drugaja venta daleka.

Ostan'tes' zdes': gotov vam užin;

V kamine razložen ogon';

Postelja est' - pokoj vam nužen,

A k stojlu tjanetsja vaš kon' “.

- „Mne putešestvie privyčno

I dnem i nočjju - byl by put', -

Tot otvečaet, - neprilično

Bojat'sja mne čego-nibud'.

Ja dvorjanin, - ni čort, ni vory

Ne mogut uderžat' menja,

Kogda spešu na službu ja “.

I don Al'fons konju dal špory,

I edet rys'ju. Pered nim

Odna idet doroga $\mathrm{v}$ gory

Uščl'em tesnym i gluxim.

Vot vyezžaet on v dolinu;

Kakuju ž vidit on kartinu?

Krugom pustynja, dič' i gol',

A v storone torčit glagol',

I na glagole tom dva tela

Visjat. Zakarkav, otletela
Alphonse enfourche son coursier;

L'aubergiste lui tient l'étrier.

"Señor, suivez mon conseil:

L'heure n'est pas de se mettre en route,

Les montagnes sont dangereuses, la nuit approche,

La venta suivante est loin.

Restez ici: le diner est prêt,

Le feu brûle dans la cheminée,

Un lit est préparé, vous avez besoin d'un

Et votre cheval sent la stalle."

repos

- "Je suis habitué de voyager jour et nuit,

Pourvu qu'il y ait un chemin-

Répond l'autre -, il ne convient pas

D'avoir peur de quoi que ce soit.

Je suis gentilhomme, ni le diable, ni les voleurs

Ne peuvent me faire obstacle

Lorsque je me presse pour reprendre $d u$ service."

Un coup d'éperons,

Don Alphonse part au trot. Devant lui,

Un chemin avance dans la montagne

Par un défilé étroit et sauvage.

Voilà quil arrive dans une vallée;

Mais que voit-il?

Le désert alentours est vide et nu;

Un gibet s'y dresse

Avec deux corps.

Une bande de corbeaux 
Vataga černaja voron,

Croassant s'envole

Liš tol'ko k nim pod"exal on.

Dès quil s'approche.

To byli trupy dvux gitanov,

C'étaient des cadavres de deux gitans.

Dvux slavnyx brat'ev-atamanov,

Deux célèbres frères-brigands,

Davno povešennyx i tam

Pendus depuis longtemps

Ostavlennyx v primer voram.

Et laissés pour instruire les voleurs.

Doždjami nebo ix močilo,

Le ciel les lavait à l'eau de pluie,

$<$ A $>$ solnce znojnoe sušilo,

Le chaud soleil les séchait,

Pustynnyj veter ix kačal,

Le vent du désert les faisait balancer

Klevat' ix voron priletal.

Et le corbeau venait leur donner son coup de

bec.

I šla molva $\mathrm{v}$ prostom narode,

Čto, obryvajas’ po nočam,

Les gens simples disaient

Que la nuit, les pendus se détachaient du

Oni do utra na svobode

Et, libres jusqu'au matin, gibet

Guljali, mstja svoim vragam.

Portaient vengeance à leurs ennemis.

Al'fonsov kon' vsxrapel i bokom

Prošel ix mimo, i potom

Le cheval d'Alphonse sébroue,

Ponessja rezvo, legkim skokom,

Les contourne avec prudence et puis

$S$ svoim besstrašnym sedokom.

Se met au galop

Portant son cavalier sans peur. 


\section{BIBLIOGRAPHIE}

\section{Textes}

Maistre, Xavier de, Voyage autour de ma chambre, Paris, Calmann Lévy, 1877.

MTS-1804 et MTS-1810 = Ротоскі, Jean, Manuscrit trouvé à Saragosse (version de 1804 et version de 1810), éd. par François Rosset, Dominique Triaire, Paris, Flammarion, 2008 (GF 1342-1343).

Ротоскі, Jan, Rękopis znaleziony w Saragossie, [Warszawa], Czytelnik, 1950, 1956, 1965 et 1976.

РотоскіJ, Jan, Rukopis', najdennaja v Saragose, Moskau, Nauka, 1968 (Serija «Literaturnye pamjatniki»).

\section{Travaux}

Beauvors, Daniel, «Jean Potocki's Voyages: From Mythic Orient to Conquered Orient", in L'Hénaurme siècle. A Miscellany of Essays on Nineteenth-Century French Literature, ed. by Will L. McLendon, Heidelberg, Carl Winter Universirätsverlag, 1984, p. 13-26.

BÈLza, Igor, "Puškin i pol'skaja kul'tura», in Pol'sko-russkie literaturnye svjazi, otv. red. N. Balasov et al., Moskau, Nauka, 1970, p. 156-174.

—, "Puškin i Jan Potockij", in Iskusstvo slova. Sbornik statej $k$ 80-letiju... D.D.Blagogo, otv. red. K. Pigarev, Moskau, Nauka, 1973, p. 125-134.

—, Portrety romantykow, Wwa, Pax, 1974.

—, "O badaniach polskiej kultury artystycznej XVIII-XIX stulecia», in Kultura polska XVIII i XIX w. i jej zwiazki z kultura Rosji. Sympozium (1978), Wroclaw/Warszawa, PAN, 1984, p. 15-30. 
Černobaev, Viktor, "K istorii nabroska "Al'fons saditsja na konja”", in Puškin: Vremennik Puškinskoj Komissii, vyp. IV-V, Moskau/ Leningrad, AN SSSR, 1939, p. 405-416.

Cornwell, Neil, "The European tradition of the "Nights": Jan Potocki and Odoevski's Russian Nights", in Fantastic currencies in comparative literature: gothic to postmodern, ed. by E. S. Shaffer, Cambridge, Cambridge University Press, 2003, p. 121140 (Comparative Criticism 24).

Dworski, Andrzej, "Jan Potocki i Puszkin", in Puszkin w kregu kultury polskiej, Slavica Wratislavensia XXX, Uniw. Wroclaw, 1983, p. 204-235; éd. russe in Puškin i pol'skaja kul'tura, SPb, Vizer, 1999.

Galon-Kurkowa, Krystyna, Dlugi zmierzch. Psychobiografia liryczna Piotra Wiazemskiego, Slavica Wratislavensia XCI, Uniw. Wroclaw, 1996.

GERŠTejn, Emma, «Lermontov i kružok šesnadcati», in Žizn' i tvorčestvo Ju.M.Lermontova, Moscou, OGIZ, 1941.

Heller, Leonid, Niqueux, Michel, Histoire de l'utopie en Russie, Paris, PUF, 1995.

Landa, Semen, "Jan Potockij i ego roman Rukopis", najdennaja v Saragose", in Jan Potockij, Rukopis', najdennaja v Saragose, Moskau, Hud. literatura, 1971, p. 5-34.

—, "Jan Potockij i ego roman...", in Jan Potockij, Rukopis' najdennajav Saragosse, Moskau, Hud. literatura, 1989, p. 5-38.

—, "Ja vižu nekij svet...", Sankt-Peterburg, 1999.

Mickiewicz, Adam, "Le cours du 15 mars 1842 », in Dziela, t. X.

Literatura slowianska. Kurs II, Czytelnik, 1955, p. 214-226.

Rosset, François, "Pourquoi Saragosse?», in De Varsovie à Saragosse: Jean Potocki et son cuvre, éd. par François Rosset, Dominique Triaire, Louvain, Peeters, 2000, p. 189-217.

—, "Roman grotesque parce que roman-somme: Le Manuscrit trouvé à Saragosse de Jean Potocki ", Colloquium Helveticum: Cahiers Suisses de Littérature Comparée, 35 (2004), p. 61-75.

Rosset, François, Triaire, Dominique (éds), De Varsovie à Saragosse. Jean Potocki et son auvre, Louvain, Peeters, 2000.

—, "Présentation. Une œuvre-vie», in Jean Potocki, Manuscrit trouvé à Saragosse (version de 1804), éd. par François Rosset, Dominique Triaire, Paris, Flammarion, 2008, p. 7-56 (GF 1342). 
SLIvkin, Evgenij, «Stixotvorenie, najdennoe v Saragosse: funkcija kategorii grammatičeskogo roda v stixotvorenii Viktora Sosnory "Ja ostavil poslednjuju pulju sebe" ", Novoe Literaturnoe Obozrenie, 93 (2008), p. 246-260.

Vel'tman, Aleksandr, Strannik, Moskau, Nauka, 1977.

Vigel', Filipp, Zapiski, t. 1, Moskau, Krug, 1928.

Żurowski, Maciej, "Le Manuscrit trouvé à Saragosse et la technique romanesque du XVIII s. ", in Jean Potocki et le Manuscrit trouvé à Saragosse. Actes du colloque organisé par le Centre de civilisation française de l'Université de Varsovie (avril 1972), Varsovie, Centre de civilisation française de l'Université de Varsovie, 1981, p. 105-138 (Les cahiers de Varsovie 3). 
